phys. stat. sol. (a) 46, 209 (1978)

Subject classification: $14.2 ; 21$

Department of Physics, The Ohio State University, Columbus ${ }^{1}$ )

\title{
The Critical Fields of Superconducting Palladium Hydride
}

\author{
By \\ D. R. Krahn, R. L. Henry, D. B. Tanner, and P. E. WigeN
}

The upper critical field of superconducting $\mathrm{PdH}_{x}(x=\mathrm{H} / \mathrm{Pd})$ is measured for a range of hydrogen concentrations $(0.88<x<0.97)$. The upper critical field is found to increase linearly with decreasing temperature over the temperature range investigated $(1.5 \mathrm{~K}<T<7.5 \mathrm{~K}$ ). The data are analyzed to obtain the critical field at zero temperature as a function of concentration. The data indicate two regimes: at low $(x<0.92)$ concentrations the upper critical field increases rapidly with concentration while for higher $(x>0.92)$ concentrations it is almost independent of concentration. It is suggested that the lower concentration $\mathbf{P d H}_{x}$ may be a mixed phase system.

Le champ critique d'en haut du supraconducteur $\mathrm{PdH}_{x}(x=\mathrm{H} / \mathrm{Pd})$ a été mesuré pour une gamme de concentrations hydrogèniques $(0,86<x<0,97)$. Le champ critique d'en haut est observée en fonction de la température entre 1,5 et $7,5 \mathrm{~K}$. Il exhibe une croissance linéaire lorsque la température se décroît. Les observations sont analysé afin d'obtenir le champ critique à $T=0 \mathrm{~K}$ en fonction de la concentration. Les observations indiquent qu'il y a denx régimes. Pour les concentrations basses $(x<0,92)$ le champ critique d'en haut croît vite avec la concentration; pour les concentrations plus haut de $x=0,92$ il est presque independent de la concentration. On suggére que pour des concentrations basses $\mathrm{PdH}_{x}$ est un système des phases mixtes.

\section{Introduction}

Since the discovery by Skośkiewicz [1] of superconductivity in the palladiumhydrogen system, the effect of magnetic fields on the superconducting state has been studied by several investigators [ 2 to 5$]$. The critical field measurements have been done for several hydrogen concentrations, $x(x=\mathrm{H} / \mathrm{Pd})$, but no single experiment covers the entire range of concentrations where superconductivity has been observed. In addition, the values obtained [ 2 to 5 ] for the upper critical field $H_{\mathrm{c} 2}$ at $T=0 \mathrm{~K}$ (extrapolated to $x=1$ ) differ by more than a factor of two. This paper describes measurements of the upper critical field of $\mathrm{PdH}_{x}$ over a wide range of concentration.

The experimental method for measuring $H_{\mathrm{c} 2}$ has varied in previous investigations. Resistance measurements as a function of applied field $[2,3]$ have given the largest values of $H_{\mathrm{c} 2}(T=0 \mathrm{~K})$ reported in the literature. Vibrating sample magnetometer [4] and flip coil measurements [5] have given lower critical fields. All experiments indicate that $H_{\mathrm{v} 2}(T=0 \mathrm{~K})$ is a constant or at best a very weak function of hydrogen concentration over the range of concentrations studied. These approximately constant values of $H_{\mathrm{c} 2}(T=0 \mathrm{~K})$ vary from $\approx 800$ to $\approx 25000 \mathrm{e}$.

When quantitatively comparing the data in the literature with the new data reported here, a problem is that investigators have used several methods to determine the hydrogen concentration for a given sample. In this work the concentration is estimated from the superconducting transition temperature in zero field, $T_{\mathrm{c}}(H=0)$. There have been several measurements which relate superconducting transition temperature $T_{c}(H=0)$ to hydrogen concentration $x$ [2,6 to 8$]$. These results have a typical spread of $1.5 \mathrm{~K}$ in $T_{\mathrm{c}}$ for a given value of $x$, or, equivalently, a 0.03 spread in $x$. The differences become even greater for $x \geqq 0.95$ and $\leqq 0.89$. In the present investigation

1) Columbus, Ohio 43210, USA.

14 physica (a) 46/1 
an average of the four sets of data referred to above was used to relate $T_{c}(H=0)$ to the concentration $x$. It is important to note that the qualitative results given here are not affected by the details of the $T_{\mathrm{c}}$ versus $x$ relationship. Also, as will be seen below, the value of $H_{\mathrm{e} 2}(T=0 \mathrm{~K})$ when extrapolated to $x=1$ is not significantly affected by variations in the concentration calibration.

\section{Experimental Techniques}

The palladium used in this experiment was a $30 \mu \mathrm{m}$ thick $99.99^{\circ}$ purity rolled foil ${ }^{2}$ ), hydrogen loading was accomplished using electrolysis in $0.1 \mathrm{~N} \mathrm{HCl}$. A current density of $200 \mathrm{~A} / \mathrm{m}^{2}$ for $50 \mathrm{~min}$ at $295 \mathrm{~K}$ brought the hydrogen concentration to $x=0.6$. Electrolysis was continued at $195 \mathrm{~K}$ to achieve $x=0.98$. This corresponds to a critical temperature of approximately $8.3 \mathrm{~K}$. Four probe de resistance measurements were made before, during, and after electrolysis. An approximate value for the hydrogen concentration can be obtained from the resistivity at a given temperature $[9,10]$. The resistivity at $77 \mathrm{~K}$ is particularly sensitive to hydrogen concentration. During electrolysis the sample was extensively cold-worked due to passage through the $\alpha-\beta$ mixed phase region of the $\mathrm{PdH}_{x}$ phase diagram. After loading and between measurements the sample was stored at $77 \mathrm{~K}$. To release hydrogen during the experiment the sample was allowed to warm rapidly to approximately $225 \mathrm{~K}$ and then was immediately returned to $77 \mathrm{~K}$ or below. Each time this was done a new (and lower) hydrogen concentration was achieved. It was noted that whenever hydrogen was released from the palladium a time period on the order of $15 \mathrm{~h}$ was required for the magnetic critical field measurements to come to equilibrium. Data taken immediately after hydrogen release process did not agree with data taken on subsequent days for a given concentration. These results suggest that a low-temperature annealing process takes place. The data presented here were all obtained after the equilibrium had been reached. A $1 \times 2.5 \mathrm{~cm}^{2}$ segment of loaded foil was rolled (at $77 \mathrm{~K}$ ) into a cylindrical shape to be mounted in the magnetometer.

A vibrating sample magnetometer ${ }^{3}$ ) was used with a $30 \mathrm{~cm}$ diameter electromagnet. The magnetic fields were measured to better than 10 Oe. Sample temperatures were measured by a calibrated gallium arsenide diode located on the sample rod approximately $2 \mathrm{~cm}$ from the sample. A temperature controller maintained a uniform temperature within $0.1 \mathrm{~K}$ during data runs. When part or all of the sample was in the superconducting state, the magnetometer measured a large moment due to supereurrents in the sample.

\section{Results}

$\mathrm{PdH}_{x}$ is a type II superconductor $[4,5]$. The magnetization, $M$, as a function of applied field, $H$, obtained in these experiments does not resemble the $M$ versus $H$ curve for a ideal type II superconductor. If the field is swept from a high $\left(H>H_{\mathrm{c} 2}\right)$ value to zero at a constant temperature, the magnetization rises from its normal state (nearly zero) value to a maximum near $H=0$, rather than going to $M=0$ at $H=0$ as is expected. For sweeps from large positive fields to large negative fields and back, the magnetization describes a hysteresis loop. If the sweep starts at $M=0, H=0$, no sharp change of magnetization is observed at any field. The measurements reported here do not give any values for the lower critical field, $H_{\mathrm{c} 1}$. The shape of the $M-I I$ loop obtained from the magnetometer is indicative of an extensively cold-worked type II superconductor [12] with a large amount of flux pinning in the sample. The

2) Materials Research Corporation, Orangeburg, New York 10962, USA.

3) Princeton Applied Research Model 155, Princeton, New Jersey 08540, USA. 
relative proportions of the loop varied for some hydrogen concentrations and temperatures. This variation is not presently understood but is probably related to the change in width of the de resistive transition reported by others $[1,2,11]$.

In the analysis of the data presented here the upper critical magnetic field $\left(H_{\mathrm{e} 2}\right)$ is defined in two different ways, each of which may be significant. As shown in the inset in Fig. 1, $H_{\mathrm{cm}}$ is defined as the maximum field at which superconductivity is observed with the magnetometer. The inset in Fig. 2 indicates that $H_{\mathrm{cb}}$ is the field at which the majority of the superconductivity is gone (with the high-field tail being neglected). Some authors [4] have suggested that $H_{\mathrm{c} \cdot \mathrm{b}}$ may be more indicative of the bulk properties of $\mathrm{PdH}_{x}$. Note from Fig. 1 and 2 that the two definitions of the critical field give the same value of $T_{\mathrm{c}}(H=0)$ but different values of $H_{\mathrm{c} 2}(T=0 \mathrm{~K})$ for each concentration of hydrogen. It is difficult to compare quantitatively critical field data taken with different experimental techniques (e.g. vibrating sample magnetometer and resistance measurements) because the definition of the critical field may depend on the output of a particular type of experiment.

Critical fields, $H_{\mathrm{cm}}$ and $H_{\mathrm{cb}}$, as a function of temperature are given in Fig. 1 and 2 , respectively, for the five hydrogen concentrations studied in this experiment. A straight line least squares fit is shown for each concentration. Extrapolation of these straight lines to zero temperature gives $H_{\mathrm{cm}}(T=0 \mathrm{~K})$ and $H_{\mathrm{cb}}(T=0 \mathrm{~K})$.

The values of $H_{\mathrm{cm}}(T=0 \mathrm{~K})$ and $H_{\mathrm{cl},}(T=0 \mathrm{~K})$ are given as a function of hydrogen concentration in Fig. 3. The symbols correspond to those on Fig. 1 and 2. The error bars along the $y$-axis are due to the probable uncertainty in the $T=0$ intercept of the least squares fit. This uncertainty is largest at the lowest concentrations due to the limited temperature range over which data was taken. Because of the uncertainity in the concentration calibration, discussed above, there may be a systematic error of up to \pm 0.01 in $x$.

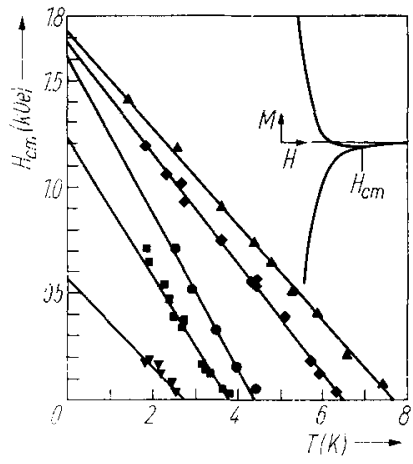

Fig. I

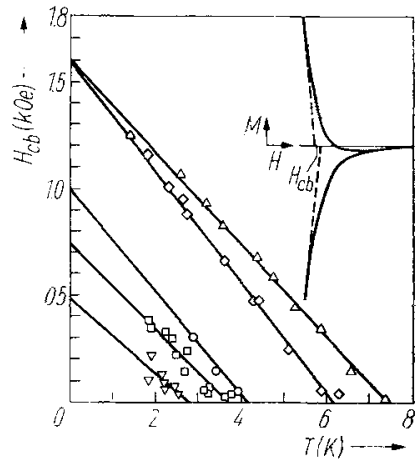

Fig. 2

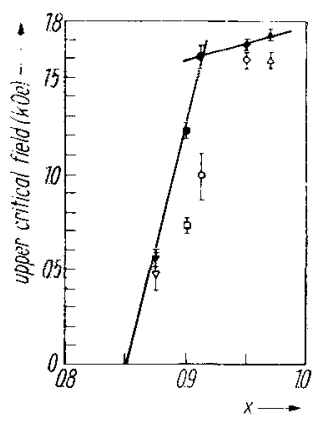

Fig. 3

Fig. 1. Upper critical field $H_{\mathrm{cm}}$, defined as indicated in the inset at upper right, versus temperature for five different hydrogen concentrations. The data are shown as points, with a different symbol for each concentration. The full lines show linear least squares fits to the data

Fig. 2. Upper critical field $H_{c b}$, defined as indicated in the inset at upper right, versus temperature for five different hydrogen concentrations. The data are shown as points, with a different symbol for each concentration. The full lines show linear least squares fits to the data

Fig. 3. Upper critical field, extrapolated to $T=0$, versus hydrogen concentration $x$. The symbols correspond to those in Fig. 1 and 2 . The hydrogen concentration was obtained from the superconducting transition temperature. The full lines are drawn as a guide to the eye 


\section{Diseussion}

The straight line fits to Fig. 1 and 2 are consistent with analysis in previous investigations $[2,3]$ although there is no theoretical prediction of this behavior. The data shown in Fig. 3 show two regimes for the $\mathrm{PdH}_{x}$ system, a constant or nearly constant value for higher concentrations and a linearly varying behavior for lower concentrations. Two straight lines have been included in Fig. 3 showing these regimes. The straight line for the lower concentrations passes through $H_{\mathrm{c}}=0$ at approximately $x=0.85$. This value of $x$ is somewhat larger than earlier observations $[2,6$ to 8$]$ when measurements are extrapolated to predict the lowest temperature at which the $\mathbf{P d H}_{x}$ system is superconducting. In the calibration of $T_{\mathrm{c}}$ versus $x$, described above, $T_{\mathrm{e}}=0$ at $x=0.79 \pm 0.02$.

Comparing Fig. 3 with earlier results [2 to 5] the values of $H_{\mathrm{em}}(T=0)$ and $H_{\mathrm{eb}}(T=$ $=0$ ) for hydrogen concentrations greater than $x=0.92$ from this study fall in the middle of the range of values obtained. It is significant that the more or less constant values of $H_{\mathrm{c} 2}(T=0 \mathrm{~K})$ obtained in earlier studies over a limited range of concentrations agree qualitatively with Fig. 3 of this work. Data of Skośkiewicz [2] show a slight downturn on the low-concentration end of $H_{\mathrm{c} 2}(T=0)$ versus $x$. This also agrees qualitatively with the present work.

Jacobs and Manchester [13] have suggested the presence of at least one new phase (the $\gamma$-phase) for the higher concentrations of hydrogen in the $\mathrm{PdH}_{x}$ system. In the low-temperature regime the turnover point in Fig. 3 at $x=0.92$ might indicate a phase boundary in the $\mathrm{PdH}_{x}$ system. It is suggested that at high concentrations of hydrogen there exists a homogeneous system. Below $x=0.92 \mathrm{PdH}_{x}$ is a mixed phase (i.e. inhomogeneous) system consisting of regions of superconductor and regions of normal metal with a lower hydrogen concentration than the superconductor. As hydrogen is removed from the bulk specimen the relative fraction of the superconductor decreases and $T_{e}$ and $H_{\mathrm{c}}$ are suppressed by proximity to normal state inclusions. The linear decrease in $H_{\mathrm{c} 2}(T=0 \mathrm{~K})$ as $x$ decreases is interpreted as a measure of the relative proportions of these two phases (just as the linear decrease in room temperature susceptibility with increasing hydrogen concentration measures the proportions of low-concentration $\alpha$-phase and higher concentration $\beta$-phase) [14].

\section{References}

[1] T. Skośkizwicz, phys. stat. sol. (a) 11, K1.23 (1972).

[2] T. Skośkiewicz, phys. stat. sol. (b) 59, 329 (1973).

[3] N. E. Alekseevskit, Yu. A. Samarskit, H. Wolf, V. I. Tsebro, and V. M. Zakosarenko, Zh. eksper. teor. Fiz., Pisma 19, 676 (1974); Soviet Phys. - J. exper. theor. Phys., Letters $19,350(1974)$.

[4] D. S. Mclachlan, T. B. Doyle, and J. P. Burger, Proc. XIV. Internat. Conf. Low Temperature Phys., Vol. 2, Otaniemi 1975 (p. 44).

[5] M. Horobiowski, T. Skośkiewicz, and E. Trojnar, phys. stat. sol. (b) 79, KI47 (1977).

[6] T. Skośkiewtez, A. W. Szafrańskt, W. Bejarowski, and B. Baranowskx, J. Phys. C 7, 2670 (1974).

[7] J. E. Shirber and C. J. M. Northrup, Jr., Phys. Rev. B 10, 3818 (1974).

[8] R. J. Miller and C. B. Satterthwaite, Phys. Rev. Letters 34, 144 (1975).

[9] A. W. Szafrański, phys. stat. sol. (a) 19, 459 (1973).

[10] J. P. Burger, D. S. MacLachlan, R. Mailfert, and B. Souffaché, Solid State Commun. $17,277(1975)$.

[11] B. Stritzker and W. Bucked, Z. Phys. 257, 1 (1972).

[12] See, for example, D. Saint-J Ames, G. Sarma, and E. J. Thomas, Type II Superconductivity, Pergamon Press, 1969 (p. 209 ff.).

[13] J. K. Jacobs and F. D. Manchester, J. less-common. Metals 49, 67 (1976).

[14] H. C. Jamieson and F. D. Manchester, J. Phys. F 2, 323 (1972).

(Received November 17, 1977) 